\title{
A study of Ni-based refractory alloys via anomalous scattering techniques
}

\author{
Michelle L. Tokarz ${ }^{\text {a) }}$ and John C. Bilello \\ Department of Materials Science and Engineering, University of Michigan, Ann Arbor, Michigan 48109 \\ and Center for Nanomaterials Science, University of Michigan, Ann Arbor, Michigan 48109
}

(Received 3 November 2005; accepted 7 July 2006; published online 5 October 2006)

\begin{abstract}
Anomalous x-ray scattering methods provided means to probe the local interactions of specific chemical pairs in a $\mathrm{Ni}-\mathrm{Nb}-\mathrm{Sn}$ sequence. Data near and far from the absorption edges of individual constituent atoms were obtained to calculate differential distribution functions, revealing the atomic arrangements. The compositional fluctuations throughout a typical $\mathrm{Ni}_{60} \mathrm{Nb}_{40-x} \mathrm{Sn}_{y}$ sample is described as alternating Ni-rich and Nb-rich clusters of $\sim 25 \AA$ dimensions. This nonrandom distribution of atomic species may partially explain the failure of previous modeling efforts of bulk metallic glasses to explain their mechanical behavior and thermal stability. (C) 2006 American Institute of Physics. [DOI: 10.1063/1.2260824]
\end{abstract}

\section{INTRODUCTION}

\section{A. Background}

Initial studies of Ni- and Zr-based bulk metallic glasses (BMGs) led to research efforts to expand this class of materials to include refractory constituents. These refractory alloy glasses (RAGs) were thought to have potential for high temperature applications. $^{1-5}$

While these materials revealed an increased thermal stability, they continue to be plagued by the same poor mechanical properties as many of the original Zr-based bulk metallic glasses, namely, brittleness. ${ }^{5,6}$ Despite the extensive studies of glassy metals to date, the fundamental understanding of the particular bonding characteristics of this class of alloys has been limited.

Many initial characterization techniques used for BMGs include neutron scattering which confirmed the overall amorphous structure as evidenced by the lack of crystalline peaks. ${ }^{1}$ Typical small angle neutron scattering (SANS) experiments, however, require large sample sizes which are typically unavailable in the research phase of a project.

Initial x-ray scattering experiments with synchrotron radiation, by contrast, revealed a through-thickness amorphous structure with an additional presence of highly strained nanoprecipitates. ${ }^{7}$ This was further confirmed with high resolution reflection mode experiments with overall crystalline portions varying from $0 \%$ to $7.5 \%$.

Mathematical processing of these data resulted in radial distribution functions (RDFs) that diverged from associated random hard sphere models. The analysis of these data was further complicated by the fact that a five-component Vitreloy-106 BMG agreed better with its corresponding model than the "perfect glass former" RAG3, ${ }^{7}$ thus requiring more advanced characterization methods, namely, anomalous $\mathrm{X}$-ray scattering.

\section{B. Anomalous x-ray scattering}

Anomalous x-ray methods are unique in that they utilize the "tunability" of a synchrotron source, in contrast with a

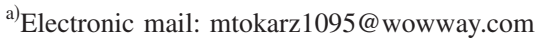

traditional fixed wavelength laboratory source. It is this tunability that allows proper selection of incident x-ray energy to maximize or minimize local bonding effects of constituent atoms, as they are expressed as reflected intensity. ${ }^{8-13}$

In order to understand anomalous x-ray scattering methods, a review of RDFs is presented according to Warren's "Approximate Methods for a Material With More Than One Kind of Atom."

Figure 1 is reproduced from Warren ${ }^{15}$ and depicts the first two steps involved in creating a RDF. First, a sum of the coherent and incoherent scattering is determined via a compositionally weighted average according to the chemical formula. Next the experimental scattering data are scaled appropriately to oscillate about this sum in order to convert an arbitrary intensity to electron units, or $I_{\text {e.u. }}$.

The difference between the scaled intensity and the coherent+incoherent sum is defined as an interference function $i(k)$,

$$
i(k)=\frac{I_{\text {e.u. }} / N-f^{2}}{f^{2}},
$$

with $\mathrm{N}$ being the total number of scattering centers and $f$ is the weighted average atomic scattering factor. Subsequently, this interference function is used for a Fourier transform to obtain an overall RDF,

$$
\begin{aligned}
\mathrm{RDF} & =4 \pi r^{2} \rho(r) \\
& =4 \pi r^{2} \rho_{0}+\frac{2 r}{\pi} \int k \times i(k) \times \sin (r k) d k .
\end{aligned}
$$

A representative RDF for a RAG4 $\left(\mathrm{Ni}_{59.35} \mathrm{Nb}_{34.45} \mathrm{Sn}_{6.2}\right)$ sample is shown in Fig. 2. This function is defined such that each local maximum corresponds to a nearest neighbor shell and the area under that peak is equal to the number of atoms in that shell, with $r$ being the distance from an average atom in angstroms.

This simplified mathematical representation, however, describes only the bonding characteristics of the average atom and does not allow one to distinguish between individual species $(\mathrm{Ni}, \mathrm{Nb}$, or $\mathrm{Sn}$ ) or constituent chemical pairs. 

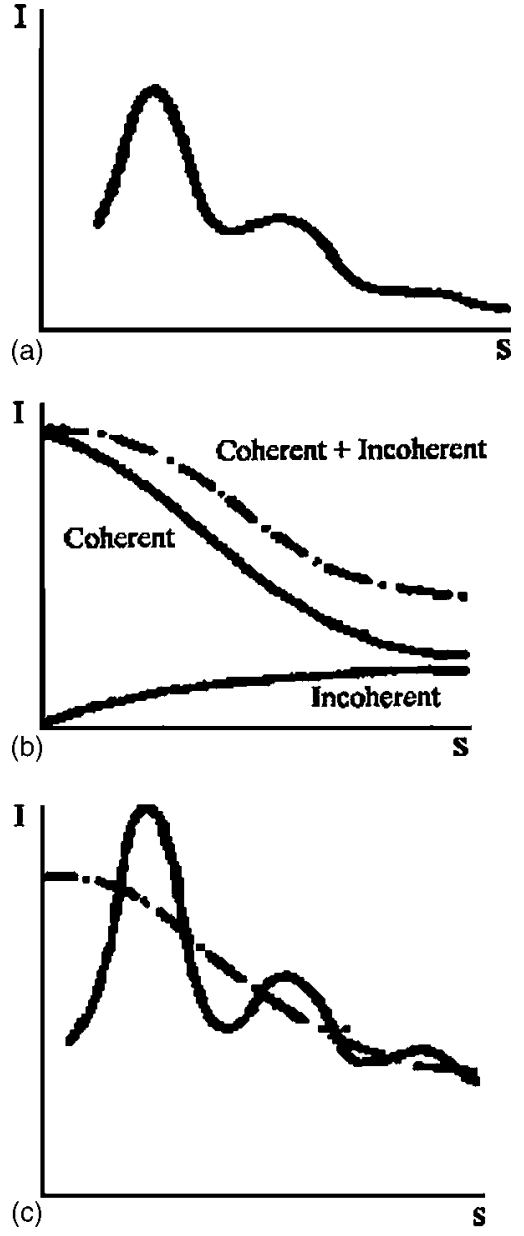

FIG. 1. Schematic of data scaling process for radial distribution analysis. Data in arbitrary units (a) are scaled to electron units by oscillation about coherent and incoherent scattering (b) to result in (c) (see Ref. 14).

(Ni-Ni, Nb-Nb, Sn-Sn, Ni-Nb, Ni-Sn, and Nb-Sn).

A careful review of the following equations shows the potential for varying atomic scattering factor corrections, $f^{\prime}$ and $f^{\prime \prime}$, in such a way as to reveal chemical behavior of individual pairs:

$$
f_{e}=\int_{0}^{\infty} 4 \pi r^{2} \rho(r) \frac{\sin (k r)}{k r} d r
$$

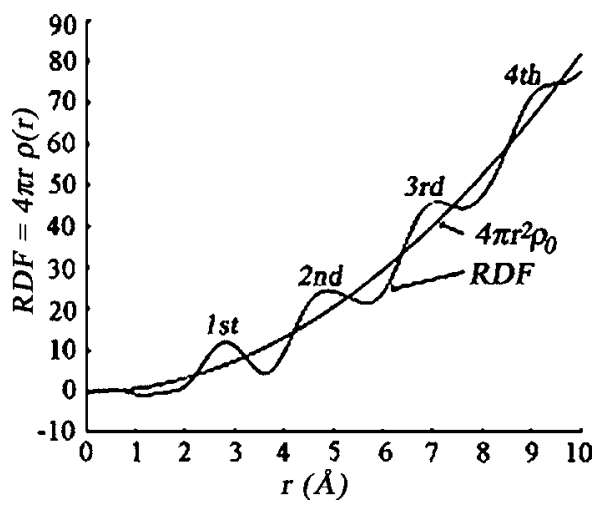

FIG. 2. Radial distribution function (RDF) for RAG4 sample. $4 \pi r^{2} \rho_{0}$ is also included for comparison. Note the presence of first four nearest neighbor shells.
$X$-ray wavelength $(\hat{A})$

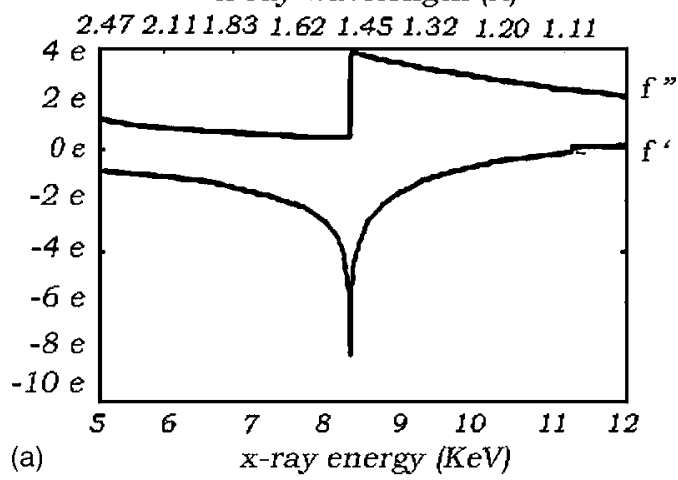

$x$-ray wavelength $(\dot{A})$

$\begin{array}{llllllllllllll}0.88 & 0.80 & 0.74 & 0.68 & 0.63 & 0.59 & 0.55 & 0.52\end{array}$

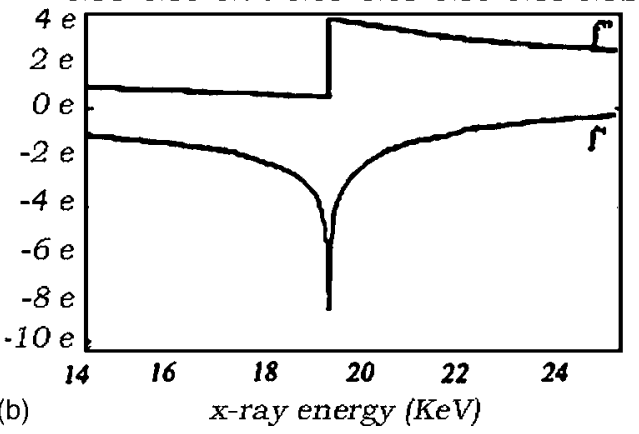

FIG. 3. Anomalous dispersion correction factors for (a) Ni edge at $8.23 \mathrm{keV}$ and (b) Nb edge at $18.99 \mathrm{keV}$.

$$
f_{a}=\sum_{n} \int_{0}^{\infty} 4 \pi r^{2} \rho_{n}(r) \frac{\sin (k r)}{k r} d r
$$

$$
f_{a}=f^{0}+f^{\prime}-i f^{\prime \prime}
$$

$$
F(s)=\sum_{n} f_{a} \exp \left(2 \pi s r_{n}\right)
$$

$$
I_{\text {e.u. }}=|F(s)|^{2},
$$

where $I_{\text {e.u. }}$ is the scattered intensity and $F(s)$ is a total structure factor. $f_{a}$ is an atomic structure factor and is related to $F$ via the cross product of the scattering vector, $s$, and atom position, $r_{n}$. Additionally, relations for the electron scattering factor, $f_{e}$, and the atomic scattering factor, $f_{a}$, are given in Eqs. (3) and (4), respectively.

Equation (5) shows the relationship between the atomic scattering factor and both anomalous corrections, $f^{\prime}$ and $f^{\prime \prime}$. $f^{0}$ is designated as the atomic scattering factor far from an absorption edge. It is important to note that at such energies, the $f^{\prime}$ and $f^{\prime \prime}$ correction factors cancel each other and become unimportant.

Figures 3(a) and 3(b) show the anomalous dispersion correction factors, $f^{\prime}$ and $f^{\prime \prime}$, for both $\mathrm{Ni}$ and $\mathrm{Nb}$ at their respective edge energies of 8.23 and $18.99 \mathrm{keV} .^{14-17}$ This edge corresponds to the energy at which the electron distribution of the particular atom deviates from a spherical shape. 


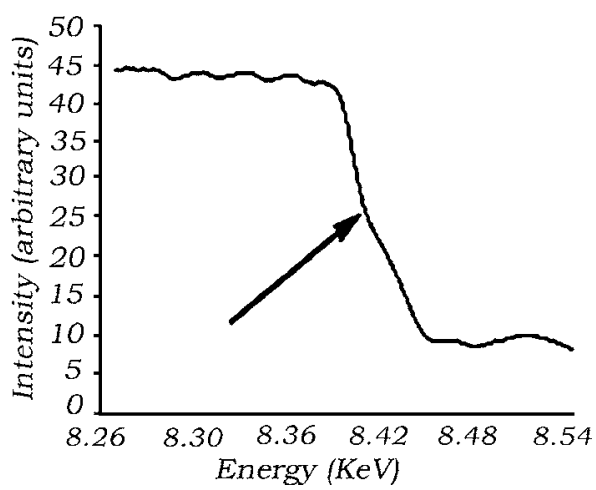

FIG. 4. Monochromator scans across Ni $K$ absorption edge. The black arrow corresponds to the point of inflection of $8223 \mathrm{eV}$.

\section{EXPERIMENTAL METHODS}

The first step in all $\mathrm{x}$-ray experiments was a monochromator scan through the edge energy with the corresponding metal foil in place. The edge energy was then calculated by finding the inflection point, as shown in Fig. 4.

This figure plots the experimental intensity versus $\mathrm{x}$-ray energy in $0.5 \mathrm{eV}$ increments with a Ni foil filter placed before the detector. The second derivative of this plot gives the inflection point that corresponds to the edge energy of $8220 \pm 0.5 \mathrm{eV}$. A similar scan with the $\mathrm{Nb}$ metal foil resulted in an edge energy of $18980 \pm 1 \mathrm{eV}$.

Once the edge energy had been unambiguously identified and calibrated with the monochromator in use, the x-ray beam was lowered in energy by $100 \mathrm{eV}$ to $8120 \pm 0.5 \mathrm{eV}$ and several scattering patterns of the refractory alloy glass sample were obtained, as shown in Fig. 5.

Subsequently, a similar process was used to identify the edge energy and calibrate the monochromator before every scattering pattern obtained at $8218.5 \pm 0.5 \mathrm{eV}$ (a difference of $5 \mathrm{eV})$.

Figure 6 shows the sum of 13 individual x-ray scattering patterns each at 8215 and $8120 \mathrm{eV}$ for a typical RAG3 sample, before the necessary radial distribution analysis corrections.

An initial review of these data reveals distinct differ-

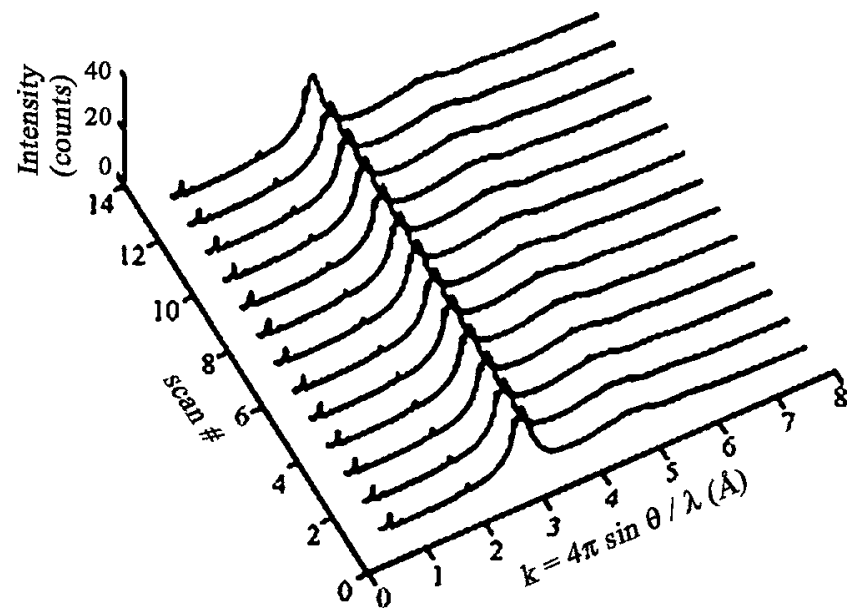

FIG. 5. Individual synchrotron scattering patterns taken for an individual RAG3 $\left(\mathrm{Ni}_{60} \mathrm{Nb}_{35} \mathrm{Sn}_{5}\right)$ sample.

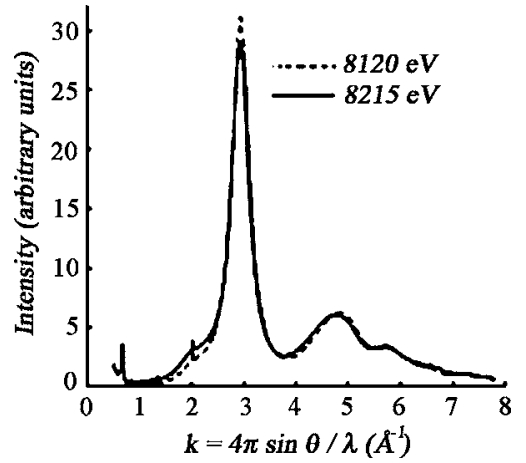

FIG. 6. Raw scattering data comparisons of a RAG3 $\left(\mathrm{Ni}_{60} \mathrm{Nb}_{35} \mathrm{Sn}_{5}\right)$ sample near $\mathrm{Ni}$ edge. Note differences in uncorrected intensities at various scattering vector, $k$, values.

ences in scattering behavior at $k \sim 2, k \sim 3$, and $k \sim 4.5$. In order to obtain quantitative results, however, several corrections must be performed.

Since the anomalous $\mathrm{x}$-ray scattering experiments are performed very close to absorption edges, fluorescence effects become important, especially for the experimental runs that are $\sim 5 \mathrm{eV}$ below the edge. For example, as one approaches $8.2 \mathrm{keV}$, the interaction between incoming $\mathrm{x}$ rays and $\mathrm{Ni}$ electrons is such that the scattered intensity function must be modified to account for the resonance that occurs. This is expressed specifically by the $f^{\prime}$ correction for phase changes and $f^{\prime \prime}$ for changes in absorption.

However, because the energies of the $K_{\beta}$ fluorescence and the elastic scattering are so similar, they are nearly inseparable. Therefore the $K_{\alpha}$ fluorescence scattering data were collected in a separate channel of the Ge detector. Then, knowing the ratio between the $K_{\alpha}$ and $K_{\beta}$ intensities, the $K_{\alpha}$ counts were subtracted to give the elastic scattering. ${ }^{16,17}$

Final corrections for multiple scattering, absorption, and polarization were performed according to Hufnagel. ${ }^{14,15}$ Once all corrections were properly made, transformations of each data set to corresponding RDFs were performed according to Eqs. (1) and (2).

Further, RDFs obtained from data $4.5 \mathrm{eV}$ from the absorption edges were designated as $\mathrm{RDF}_{\text {near }}$ and those $100 \mathrm{eV}$ from the edge were designated as $\mathrm{RDF}_{\text {far }}$.

Final mathematical manipulation of RDF results was, according to Eq. (8), as follows:

$$
\mathrm{DDF}=\mathrm{RDF}_{\text {far }}-\mathrm{RDF}_{\text {near }},
$$

where DDF is defined as a differential distribution function.

Noting the contributions of the anomalous dispersion correction factors, $f^{\prime}$ and $f^{\prime \prime}$, from Eqs. (3)-(7), it is apparent that $\mathrm{x}$-ray scattering intensity for a particular atomic species will be minimal at the edge energy. In addition, this intensity will increase as the incident energy is decreased, leading to the following relation:

$$
\mathrm{RDF}_{\text {far }}>\mathrm{RDF}_{\text {near }} \text {. }
$$

The DDF data were examined in detail as outlined in the Results section. 


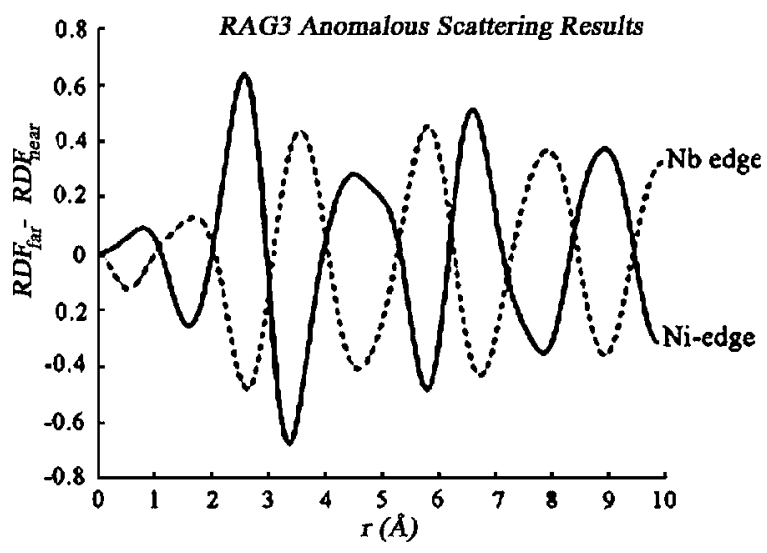

FIG. 7. DDF comparisons for RAG3 $\left(\mathrm{Ni}_{60} \mathrm{Nb}_{35} \mathrm{Sn}_{5}\right)$.

\section{RESULTS}

Figure 7 compares DDFs for the RAG3 composition $\left(\mathrm{Ni}_{60} \mathrm{Nb}_{35} \mathrm{Sn}_{5}\right)$ for data obtained at both the $\mathrm{Ni}$ and $\mathrm{Nb}$ edges. In general, it is noted that an increase in the DDF function for the Ni edge is associated with a corresponding decrease for the $\mathrm{Nb}$ edge and vice versa. ${ }^{18-23}$

This is an indication that any tendency for $\mathrm{Ni}$ association (for example, Ni-Ni bonding) is compensated for by a decrease in $\mathrm{Nb}$ association. Given the fact that these ternary $\mathrm{Ni}-\mathrm{Nb}-\mathrm{Sn}$ materials are nominally $5 \% \mathrm{Sn}$, this gives evidence of an alternating $\mathrm{Ni}$-rich/Nb-rich cluster model.

To investigate this phenomenon in more detail, DDF data for both edges were extended to $\sim 40 \AA$ and is shown in Figs. 8(a) and 8(b). In both of these figures, there is a general damping of oscillations to approximately $25 \AA$, corresponding to a cluster size of that dimension.

In order to construct a complete physical model that takes into account the cluster sizes, the chemical composition was used to calculate a bcc-like structure, as shown in Fig. 9.

It is the 1:1 correspondence between Ni-enriched clusters and $\mathrm{Nb}$-enriched clusters of similar sizes that gives credibility to a body-centered-cubic structure. Additionally, given the correct amount of "enrichment" in any cluster, adjustments can be made and compared to overall chemical compositions of these materials.

\section{DISCUSSION}

\section{A. Additional observations}

DDFs for both Ni-edge and $\mathrm{Nb}$-edge scattering data reveal cluster sizes of $\sim 25 \AA$. Additionally, the opposing behavior of these functions suggests an alternating structure, as described pictorially in the bcc model in Fig. 9.

There are several other observations about this particular class of materials which can now be understood with a different perspective.

Previous thermal stability work shows substantial differences between elevated temperature behavior as indicated by bulk measurements such as differential scanning calorimetry (DSC), and local measurements such as in situ x-ray diffraction. Figure 10 shows the specific example of local crystallization occurring at $460{ }^{\circ} \mathrm{C}, 140^{\circ}$ below the $T_{g}$.
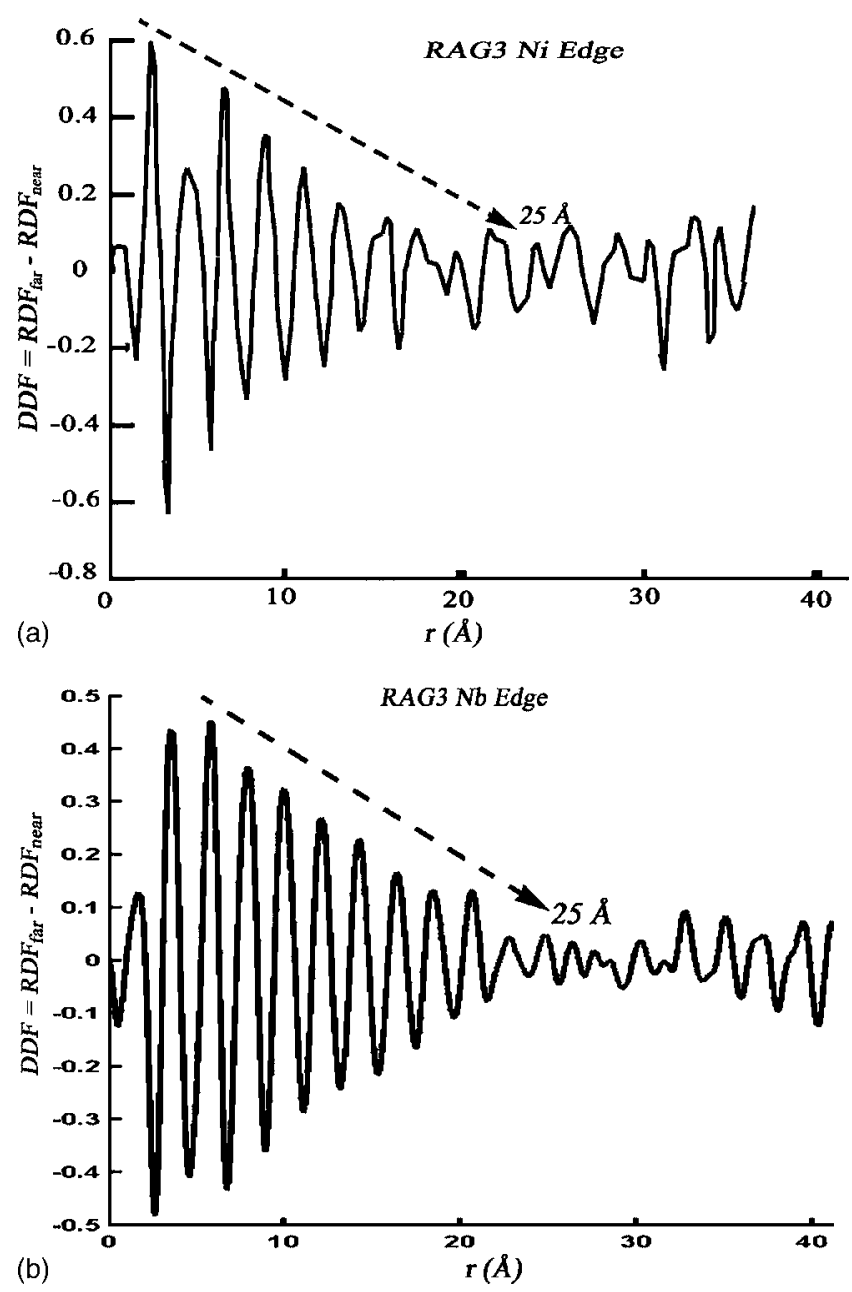

FIG. 8. Differential RDF plot for a typical RAG3 $\left(\mathrm{Ni}_{60} \mathrm{Sn}_{35} \mathrm{Sn}_{5}\right)$ sample extrapolated to $40 \AA$.

Figures 11(a) and 11(b) are scanning electron microscopy (SEM) images of a polished surface of a RAG1 $\left(\mathrm{Ni}_{60} \mathrm{Nb}_{37} \mathrm{Sn}_{3}\right)$ sample. Two typical characteristics of this series of refractory alloy glass samples are immediately obvious from these figures. The first is the brittle behavior as manifested by the inability to create a smooth surface with standard sample polishing methods. This is especially obvious in Fig. 11(b) where several triangular features cover the surface. Secondly, several micron sized precipitates are distributed throughout this particular sample.

\section{B. Comparisons to other BMGs}

These observations are best understood by making analogies to a well-known bulk metallic glass, Vitreloy-106 $\left(\mathrm{Zr}_{57} \mathrm{Nb}_{5} \mathrm{Cu}_{15.4} \mathrm{Ni}_{12.6} \mathrm{Al}_{10}\right)$. This is traditionally processed via a melt-injection method with temperatures of $700-900{ }^{\circ} \mathrm{C}$ under an $\mathrm{Ar}$ inert atmosphere and results in fully amorphous bulk alloys consistently.

By contrast, when this same method is used to process refractory BMGs that include a significant amount of $\mathrm{Nb}$ $\left(T_{m}=2477{ }^{\circ} \mathrm{C}\right)$, a persistent amount of oxide $\left(\mathrm{Nb}_{2} \mathrm{O}_{5}\right)$ and intermetallic $\left(\mathrm{Ni}_{3} \mathrm{Sn}\right)$ nanocrystalline residuals remains in the as-cast samples. ${ }^{6}$ 
(a)

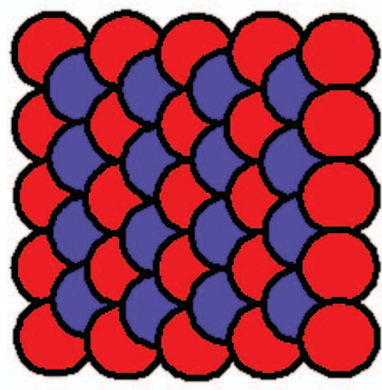

(b)

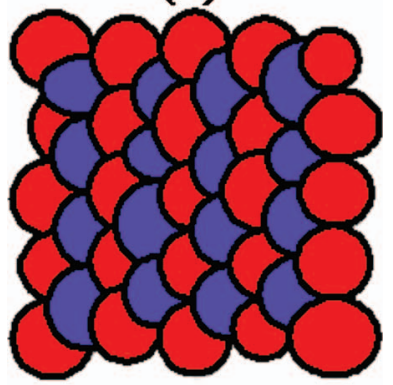

(c)

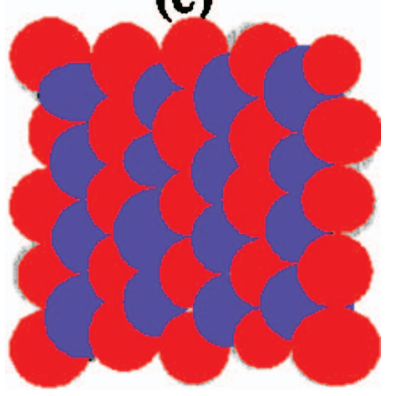

FIG. 9. (Color) bcc-like model for cluster arrangement of RAG3 $\left(\mathrm{Ni}_{60} \mathrm{Nb}_{35} \mathrm{Sn}_{5}\right)$ alloy consisting of Ni-rich (blue spheres) and $\mathrm{Nb}$-rich clusters. Beginning with distinct clusters of $25 \AA$ radii (a), this model is slightly perturbed to reveal variations from this distance (b). Finally, the abrupt transition from a Ni-rich region to a $\mathrm{Nb}$-rich region is softened to reveal nondistinct edges to these clusters in (c).

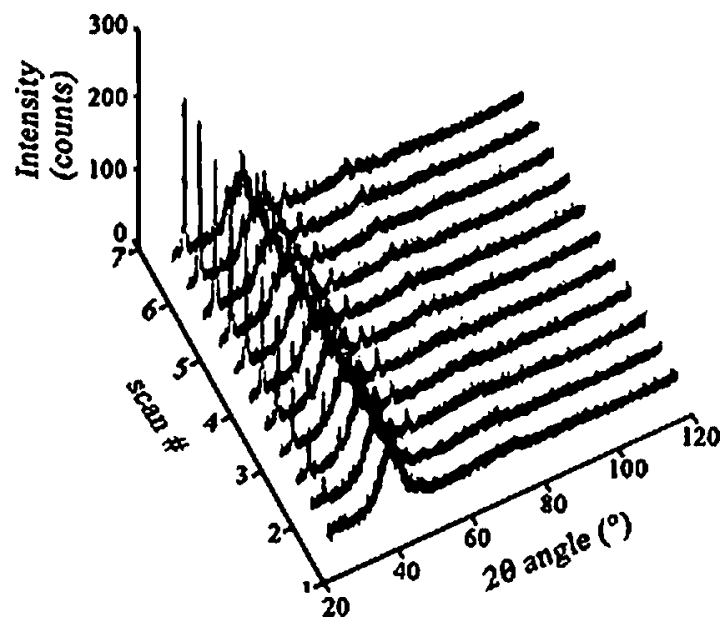

FIG. 10. In situ $\mathrm{x}$-ray scans with heating of RAG3 at $460{ }^{\circ} \mathrm{C}$ (scan time $\sim 90$ min.).
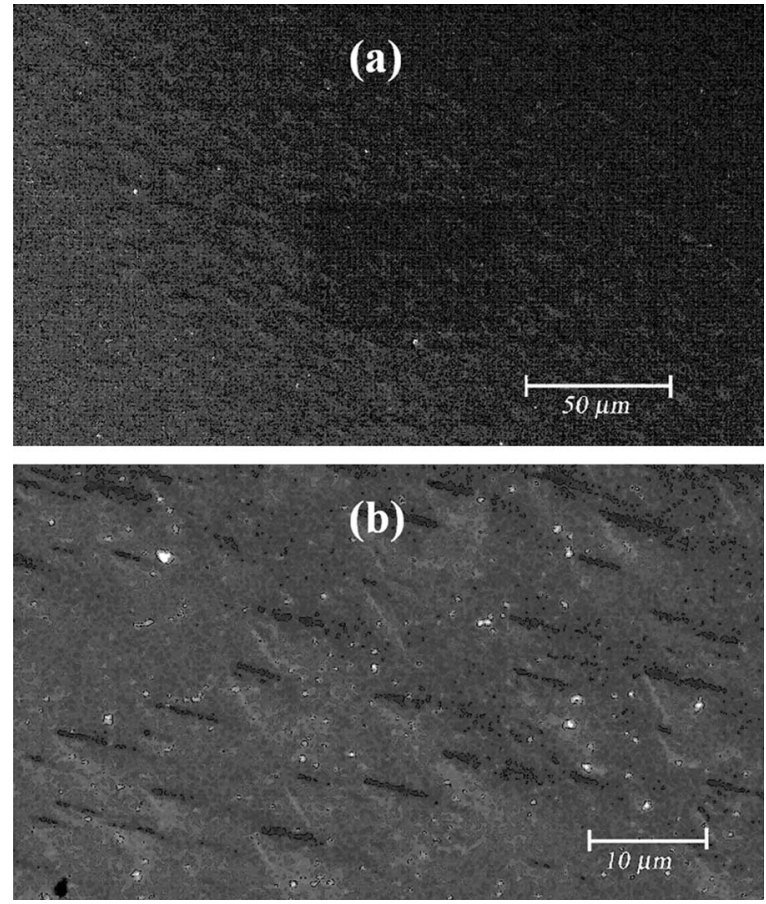

FIG. 11. [(a) and (b)] SEM images of polished surface. Note raised features of micron dimensions.

These precipitates serve as nucleation sites for further crystallization at temperatures below the glass transition temperature $T_{g}$.

Previous work on bulk metallic glasses also shows a greater agreement with a random hard sphere model for the five-component Vitreloy, as compared to the RAG3 composition. The affinity between $\mathrm{Ni}$ and $\mathrm{Sn}$, thus forming $\mathrm{Ni}$ enhanced clusters, partially explains this phenomenon. Additionally, $\mathrm{Nb}$ being a well-known oxide former may also form such $\mathrm{Nb}$-enhanced clusters. There is no such known mechanism for cluster formation in the Vitreloy-106 system.

\section{CONCLUSIONS}

Using synchrotron techniques, correlations between atomic specific bonding characteristics and thermal behavior were possible. Previous research with hard sphere models and standard RDF analyses revealed a nonrandom clustering effect that was not feasible to investigate with laboratory $\mathrm{X}$-ray sources, thus prompting the use of more advanced synchrotron techniques.

Utilizing the focusing capabilities of a typical synchrotron beamline in conjunction with a solid state Ge detector, these sophisticated methods allowed the calculation of differential distribution functions (DDFs) as initially outlined by Hufnagel. ${ }^{15,16}$ These DDFs revealed cluster sizes on the order of $25 \AA$ for both $\mathrm{Ni}$-edge and $\mathrm{Nb}$-edge experiments, thus leading to a bcc model of alternating Ni-rich and $\mathrm{Nb}$-rich clusters.

These nonrandom fluctuations in $\mathrm{Ni}$ and $\mathrm{Nb}$ associated bonding also help us explain previous stability studies that showed a fundamental difference between refractory alloy glasses (RAGs) and more traditional BMGs as manifested both in bulk thermal behavior and in localized onset of crys- 
tallization. These results also give additional insight regarding nucleation and growth mechanisms occurring during typical injection mold processing of bulk metallic glasses.

\section{ACKNOWLEDGMENTS}

Research supported by DARPA under Contract No: DAAD19-01-1-0525 via a subcontract from California Institute of Technology and also the Army Research Office under Contract No. DAAD19-02-0335. Portions of this research were carried out at the Stanford Synchrotron Radiation Laboratory, a national user facility operated by Stanford University on behalf of the U.S. Department of Energy, Office of Basic Energy Sciences. Additionally, the assistance of Zofia Rek and Apurva Mehta was invaluable in the use of several beamlines. Finally, we are especially thankful to Sean Brennan and Todd Hufnagel who graciously provided not only specific MATLAB programming routines and equipment setup but also an enormous amount of encouragement.

${ }^{1}$ W. L. Johnson, S. J. Poon, J. Durand, and P. Duwez, Phys. Rev. B 18, 206 (1978).

${ }^{2}$ P. Duwez, R. H. Willens, and R. C. Crewdson, J. Appl. Phys. 36, 2267 (1965).

${ }^{3}$ A. Peker and W. L. Johnson, Appl. Phys. Lett. 63, 2342 (1993).
${ }^{4}$ A. Masuhr, T. A. Waniuk, R. Busch, and W. L. Johnson, Phys. Rev. Lett. 82, 2290 (1999).

${ }^{5}$ M. L. Tokarz, Ph.D. thesis, University of Michigan, 2004.

${ }^{6}$ M. L. Tokarz, S. A. Speakman, W. D. Porter, and J. C. Bilello, J. NonCryst. Solids 352, 3236 (2004).

${ }^{7}$ M. L. Tokarz, M. Daniels, J. C. Bilello, and Z. Rek, Mater. Res. Soc. Symp. Proc. 754, CC2.30 (2003).

${ }^{8}$ M. L. Tokarz and J. C. Bilello, Mater. Res. Soc. Symp. Proc. 754, MM9.5 (2004).

${ }^{9}$ F. Bettis, A. Bienenstock, and C. W. Bates, Jr., J. Non-Cryst. Solids 8-10, 364 (1972)

${ }^{10}$ P. H. Fuoss, Ph.D. thesis, Stanford University, 1980.

${ }^{11}$ P. H. Fuoss, W. K. Warburton, and A. Bienenstock, J. Non-Cryst. Solids 35-36, 1233 (1980).

${ }^{12}$ J. Kortright and A. Bienenstock, J. Non-Cryst. Solids 61-62, 273 (1984).

${ }^{13}$ A. Bienenstock, J. Non-Cryst. Solids 106, 17 (1988).

${ }^{14}$ B. E. Warren, X-ray Diffraction (Dover, New York, 1969), pp. 116-142.

${ }^{15}$ T. C. Hufnagel, Ph.D. thesis, Stanford University, 1995.

${ }^{16}$ T. C. Hufnagel, S. Brennan, P. Zschack, and B. M. Clemens, Phys. Rev. B 53, 12024 (1996).

${ }^{17}$ S. Brennan, Rev. Sci. Instrum. 63, 992 (1992).

${ }^{18}$ S. Brennan and P. L. Cowan, Rev. Sci. Instrum. 63, 850 (1992).

${ }^{19}$ R. G. Munro, Phys. Rev. B 25, 5037 (1982).

${ }^{20}$ N. J. Shevchik, Philos. Mag. 35, 805 (1977).

${ }^{21}$ N. J. Shevchik, Philos. Mag. 35, 1289 (1977).

${ }^{22}$ L. Kissel and R. H. Pratt, Acta Crystallogr., Sect. A: Found. Crystallogr. A46, 1537 (1990).

${ }^{23}$ B. E. Warren and G. Mavel, Rev. Sci. Instrum. 36, 196 (1965). 